\title{
Association between the SLC6A4 gene and schizophrenia: an updated meta-analysis
}

This article was published in the following Dove Medical Press journal:

Neuropsychiatric Disease and Treatment

\section{Feng-ling $\mathrm{Xu}$ \\ Bao-jie Wang \\ Jun Yao}

School of Forensic Medicine, China Medical University, Shenyang IIOI22, People's Republic of China
Correspondence: Bao-jie Wang; Jun Yao School of Forensic Medicine, China Medical University, No 77 Puhe Road, Shenbei New District, Shenyang I I0I22, People's Republic of China

Tel +862431939433

Email wangbj77@।63.com; yaojun198717@163.com
Background: In order to explore the association between the SLC6A4 gene and the risk of schizophrenia, an updated meta-analysis was conducted using a total of 46 scientific articles. Methods: Through a literature search, papers studied included 35 articles on serotonin-transporter-linked polymorphic region (5-HTTLPR) with 8,752 cases and 10,610 controls, 17 articles on second intron variable number of tandem repeats with 7,284 cases and 8,544 controls, four studies on rs1042173 with 1,351 cases and 2,101 controls, and four studies on rs140700 with 1,770 cases and 2,386 controls. Pooled, subgroup, and sensitivity analyses were performed, and the results were visualized by forest and funnel plots.

Results: An association between 5-HTTLPR and the risk of schizophrenia was not found, except for an Indian subgroup analysis $(P=0.014, \mathrm{OR}=1.749,95 \% \mathrm{CI}=1.120-2.731)$. A 10 repeats $/ 12$ repeats $(10 \mathrm{R} / 12 \mathrm{R})$ genotype was a protective factor against schizophrenia $\left(P_{z}=0.020, \mathrm{OR}=0.789\right.$, $95 \% \mathrm{CI}=0.646-0.963)$, but a $12 \mathrm{R} / 12 \mathrm{R}$ genotype was a risk factor for schizophrenia $\left(P_{z}=0.004\right.$, $\mathrm{OR}=1.936,95 \% \mathrm{CI}=1.238-3.029)$ in the pooled analyses. In Caucasians, a GG genotype of rs 1042173 may be a risk factor for schizophrenia $\left(P_{z}=0.006, \mathrm{OR}=1.299,95 \% \mathrm{CI}=1.079-1.565\right)$. No association was found between rs140700 and the risk for schizophrenia.

Conclusion: Through meta-analysis, we were able to gain insight into previously reported associations between SLC6A4 polymorphism and schizophrenia.

Keywords: SLC6A4, gene, schizophrenia, meta-analysis, polymorphism

\section{Introduction}

Schizophrenia is a complex chronic brain dysfunction that has an elusive pathogenesis and is highly heritable. ${ }^{1}$ Investigations into twins and adoptees have shown that schizophrenia was caused by both genetic and environmental factors. ${ }^{2,3}$ Epidemiological genetic studies suggested that genetic factors contributed significantly to the etiology of schizophrenia. ${ }^{4}$ Pathological mechanisms are based on various neurotransmitter and neurodevelopmental hypotheses, and the hypothesis of a 5-hydroxytryptamine (5-HT) system defect is an important one. The serotoninergic pathway has been implicated, for several reasons, as having a major role in the pathophysiology of schizophrenia. By binding with receptors, 5-HT negatively regulates cAMP-dependent signal transduction and inhibits neuronal activity by opening G-protein-gated inwardly rectifying potassium channels..$^{5}$ The serotonin transporter (5-HTT) has a crucial function in the regulation of 5-HT reuptake in presynaptic neurons. It has been noted that levels of 5-HTT change in schizophrenic patients. ${ }^{6,7}$ Significant differences in mRNA levels of the serotonin transporter gene $(S L C 6 A 4)^{8}$ and serotonin transporter protein levels ${ }^{9}$ were observed in schizophrenic patients compared with healthy controls. Pharmacological evidence indicated that 5-HTT was a site of action for several drugs with central nervous system effects ${ }^{10,11}$ and that 5-HTT was involved in the pathogenesis of schizophrenia. ${ }^{12,13}$ 
Therefore, the SLC6A4 gene is a candidate gene for the pathogenesis of schizophrenia.

The most studied polymorphisms in the SLC6A4 gene are a 44-base pair (bp) insertion-deletion (serotonin-transporterlinked polymorphic region [5-HTTLPR]) in the promoter region, generating major $\mathrm{L}$ and $\mathrm{S}$ alleles, and a 17-bp variable number of tandem repeats (VNTR) in the second intron (STin2). ${ }^{14}$ The STin2 consists of 17-bp VNTR elements existing in 9,10 , and 12 repeats (9R, 10R, and 12R), although other rare types, such as seven-repeat units, have also been reported. The single-nucleotide polymorphisms (SNPs), rs1042173 and rs140700, are located in the three prime untranslated region and intron 5 of the SLC6A4 gene, respectively. Associations between the SLC6A4 gene and schizophrenia are controversial. ${ }^{15,16}$ Ambiguous results from different studies may possibly reflect sample sizes insufficient for obtaining adequate statistical power.

A meta-analysis is a useful method for interpreting controversial study results. ${ }^{17,18}$ Four meta-analyses of the association between the SLC6A4 gene and schizophrenia have been conducted; ${ }^{19-22}$ however, the results are still controversial. In addition, a meta-analysis of the association between schizophrenia and rs1042173 and rs140700 does not exist. Thus, we intended to perform an updated meta-analysis to better analyze the association of SLC6A4 with schizophrenia.

\section{Materials and methods Literature searches}

To identify studies eligible for inclusion in this meta-analysis, English (PubMed and SchizophreniaGene [SzGene]) and Chinese language (China National Knowledge Infrastructure, Wanfang, and Weipu) databases were searched using the following keywords: "serotonin transporter," "SERTPR," "SERT-in2," "5-HTTLPR," "STin2 VNTR," "SLC6A4," and "schizophrenia." References of the searched articles were also reviewed to uncover more data.

\section{Inclusion and exclusion criteria}

Studies included in the meta-analysis met the following criteria: 1) case-control design; 2) involved patients with schizophrenia; 3) presented relevant data for case and control groups (eg, allele/genotype frequencies, sample size, ethnicity, schizophrenia diagnostic criteria, and control group source); 4) removed duplicate sample data; and 5) published before September 2018. If the article did not contain detailed data, we e-mailed the authors for further information. Studies were excluded for the following reasons: 1) family-based studies; 2) no control group; 3) no usable genotype frequency data (attempts were made to contact authors via e-mail for such data); and 4) duplicate reported sample data.

\section{Statistical analyses}

The meta-analysis was conducted using Stata Version 10.0 (Stata Corp., College Station, TX, USA). A $P$-value of Hardy-Weinberg equilibrium $\left(P_{\mathrm{HWE}}\right)$ was calculated for control groups. Associations between SLC6A4 and the risk of schizophrenia were detected under the random model..$^{23,24}$ A suitable genetic model was selected according to the previous articles. ${ }^{25}$ ORs and $95 \%$ CIs were calculated in the pooled and subgroup analyses.

The heterogeneity of studies was determined by using Cochran's chi-squared $Q$-statistic test. ${ }^{26}$ The degree of heterogeneity was expressed as $I^{2}$, which was divided into low $\left(I^{2}<25 \%\right)$, medium $\left(I^{2} \sim 50 \%\right)$, and high $\left(I^{2}>75 \%\right)$ heterogeneity. ${ }^{27,28}$ Publication bias was calculated by using Egger's test and was visualized in a funnel plot, in which the SE of the $\log$ OR of each study was plotted against its log OR. Sensitivity analysis, by removing one single study in turn, was conducted to test the impact of each study on pooled results. $P$-values of association, heterogeneity, and publication bias tests were represented by $P_{z}, P_{h}$, and $P_{e}$, respectively. In this study, $P<0.05$ was regarded as statistically significant in all statistical tests. ${ }^{29}$ Statistical power was calculated by a PS program (Adobe Systems Incorporated, San Jose, CA, USA). ${ }^{25}$

\section{Results \\ Description of studies}

A total of 380 English and 16 Chinese published research articles were searched, and 46 articles were analyzed in this study after exclusion according to a PRISMA flow program (Figure 1). ${ }^{30}$ Detailed data on five articles could not be obtained after sending e-mails to the authors; ${ }^{31-35}$ therefore, they were removed in the present meta-analysis. Table 1 describes the baseline characteristics of 46 studies that were included in this meta-analysis. The studies included 35 articles

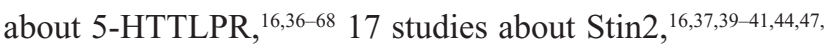
53,59,65,69-75 four articles about rs $1042173,,^{40,47,76,77}$ and four articles about rs $140700 . .^{71,76,78,79}$

\section{No association between 5-HTTLPR and the risk of schizophrenia}

In a random model ${ }^{80,81}$ the pooled and subgroup analyses of 8,752 cases and 10,610 controls were performed (Table 2). Table 3 summarizes the results of the pooled analyses 


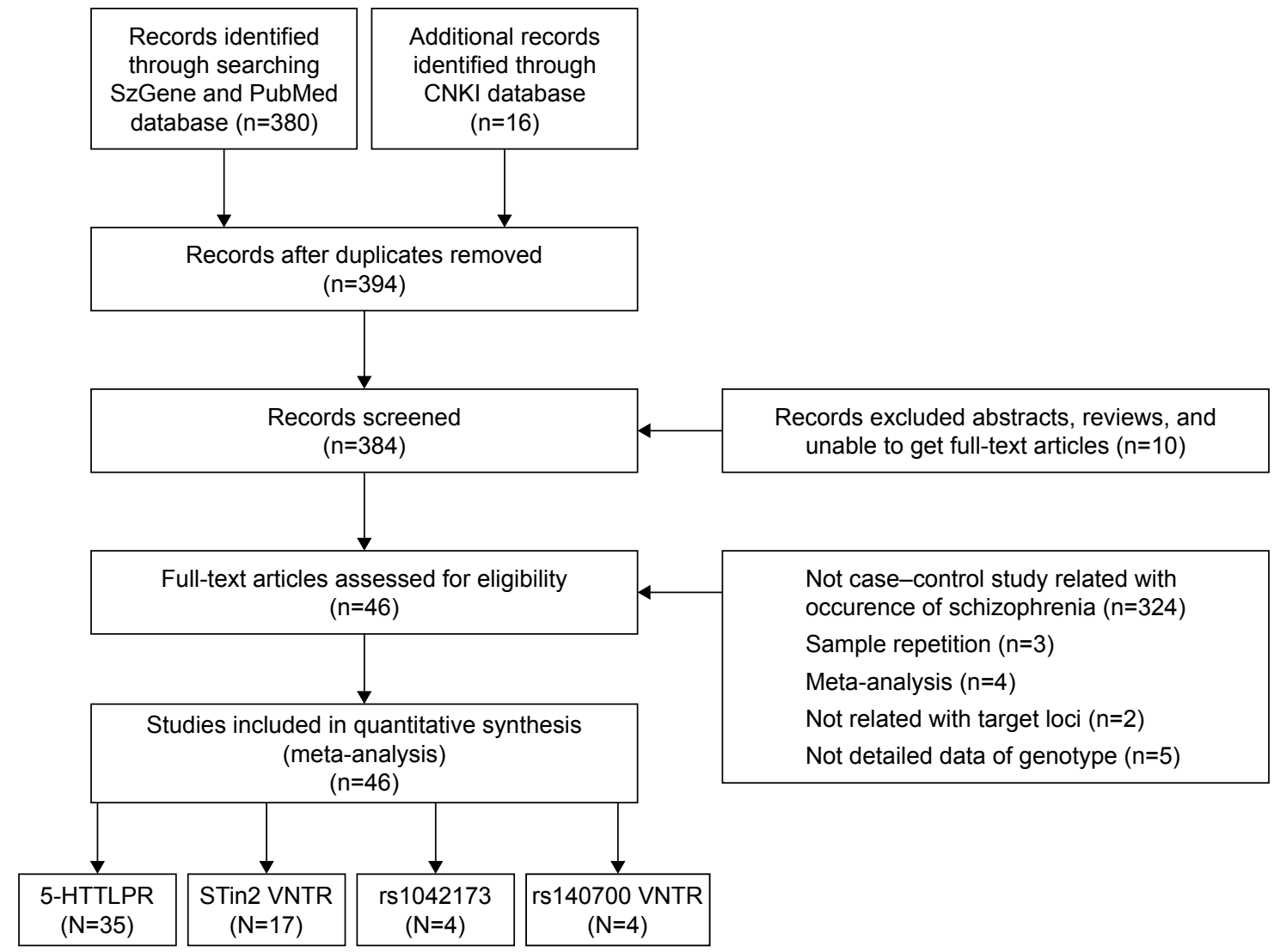

Figure I Study selection process in this meta-analysis.

Abbreviations: CNKI, China National Knowledge Infrastructure; 5-HTTLPR, serotonin-transporter-linked polymorphic region; STin2 VNTR, second intron variable number of tandem repeats.

and Table 4 depicts the data from the subgroup analyses. No association was found between 5-HTTLPR and the risk of schizophrenia with $P_{z}=0.054(\mathrm{OR}=1.085,95 \%$ $\mathrm{CI}=0.999-1.178)$ with a power of 0.935 in the dominant model. ${ }^{82}$ No associations were found in the subgroup analyses, except in an Indian group $\left(P_{z}=0.014, \mathrm{OR}=1.749\right.$, $95 \%$ CI $=1.120-2.731)$. No significant heterogeneity was observed in the pooled analysis $\left(P_{h}=0.294, P^{2}=10.4 \%\right)$.

\section{Genotypes I0R/I2R and I2R/I2R of STin2 VNTR may be associated with the risk of schizophrenia}

The allele frequencies of 7,284 cases and 8,544 controls were included in the pooled and subgroup analyses, under a random model (Table 5); 10R and 12R are common alleles; therefore, alleles $(10 R$ and $12 R)$ and genotypes $(10 R / 10 R$, $10 R / 12 R$, and $12 R / 12 R$ ) were analyzed for an association with the risk of schizophrenia, respectively (Tables 3 and 4). A $10 \mathrm{R} / 12 \mathrm{R}$ genotype was a protective factor against schizophrenia $\left(P_{z}=0.020, \mathrm{OR}=0.789,95 \% \mathrm{CI}=0.646-0.963\right)$, but a $12 \mathrm{R} / 12 \mathrm{R}$ genotype was a risk factor for schizophrenia
$\left(P_{z}=0.004, \mathrm{OR}=1.936,95 \% \mathrm{CI}=1.238-3.029\right)$ in the pooled analyses. The two pooled analyses had high powers of 1.000. In the subgroup analyses, $10 \mathrm{R} / 12 \mathrm{R}$ was a protective factor against schizophrenia in East Asia $\left(P_{z}=0.040\right.$, OR $=0.617,95 \%$ CI $=0.389-0.978)$, India $\left(P_{z}=0.014\right.$, $\mathrm{OR}=0.635,95 \% \mathrm{CI}=0.441-0.913)$ and in a population-based analysis $\left(P_{z}=0.028, \mathrm{OR}=0.794,95 \% \mathrm{CI}=0.646-0.976\right)$. A $12 \mathrm{R} / 12 \mathrm{R}$ genotype was a risk factor for schizophrenia in East Asia $\left(P_{z}=0.000, \mathrm{OR}=4.482,95 \% \mathrm{CI}=2.312-8.689\right)$ and in population-based $\left(P_{z}=0.013, \mathrm{OR}=1.755,95 \%\right.$ $\mathrm{CI}=1.124-2.742)$ and hospital-based $\left(P_{z}=0.000, \mathrm{OR}=10.689\right.$, $95 \% \mathrm{CI}=5.303-21.544)$ subgroup analyses. The significant heterogeneity was observed in these associated analyses.

\section{Genotype GG of rs 1042 I 73 may be a risk factor for schizophrenia in Caucasians}

In a recessive and a random model, no association was detected among 1,351 cases and 2,101 controls $\left(P_{z}=0.057\right.$, $\mathrm{OR}=1.199,95 \% \mathrm{CI}=0.994-1.445 ;$ Table 6 ). Tables 3 and 4 shows the results. In Caucasians, a GG genotype may be a 


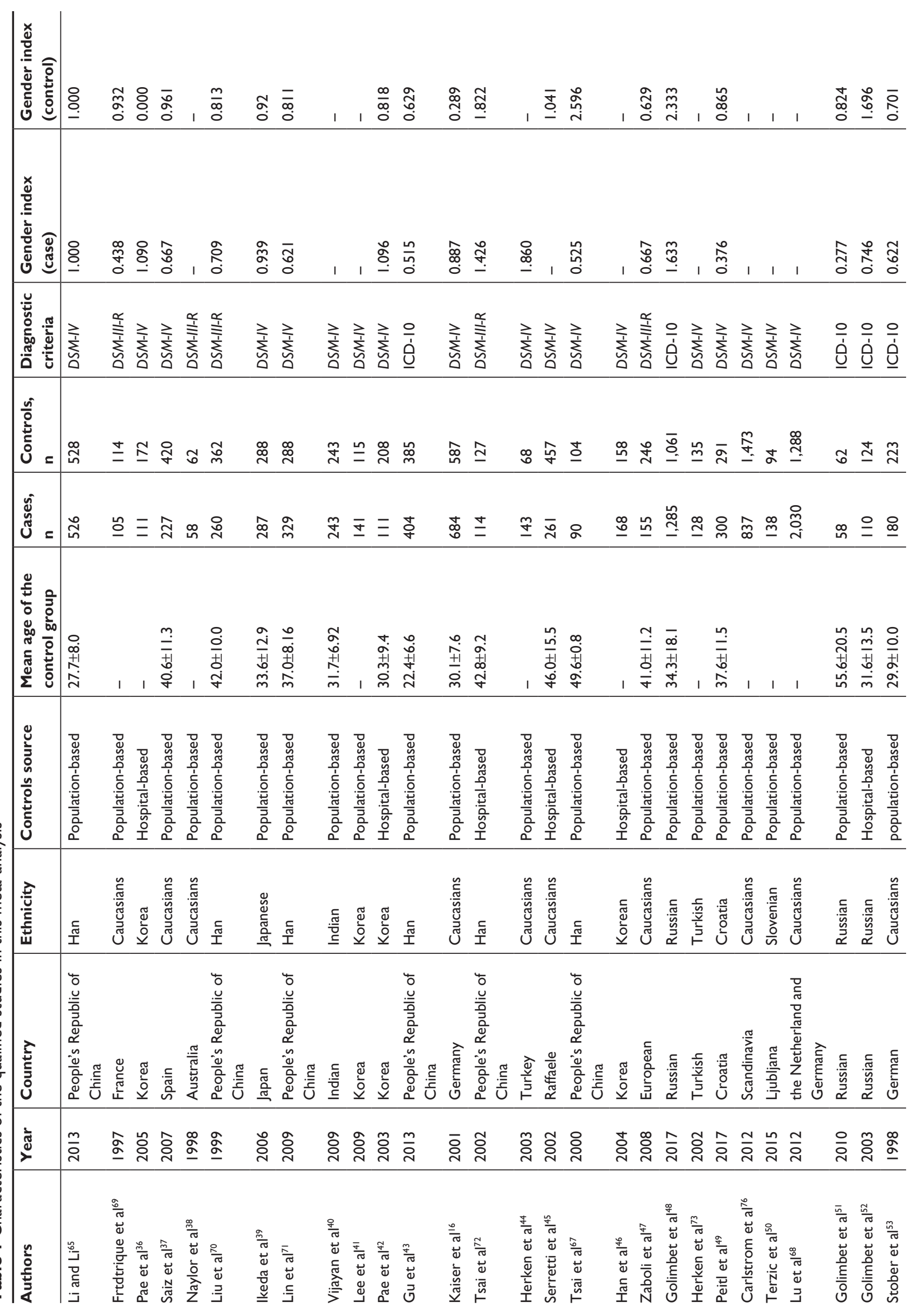




\begin{tabular}{|c|c|c|c|c|c|c|c|c|c|c|c|c|c|c|}
\hline $1 \stackrel{\bar{\sigma}}{-} \stackrel{m}{\underline{\alpha}} \frac{m}{\underline{m}}$ & 宮 & & 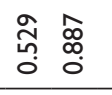 & 1 & I & 7 & । & $\begin{array}{c}\alpha \\
\alpha \\
\alpha \\
c\end{array}$ & & సે & 1 & & & ¿̊. \\
\hline 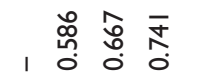 & $\underset{0}{+}$ & & 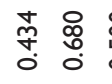 & $\begin{array}{l}8 \\
\text { iñ } \\
0\end{array}$ & & & & $\stackrel{\check{\alpha}}{c}$ & & $\frac{O}{m}$ & I & & s. & هั \\
\hline 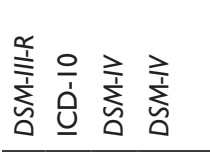 & 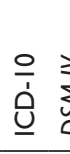 & & 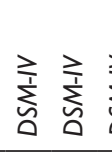 & $\sum_{\text {\} }^{i}} &{ } &{ } &{\text { 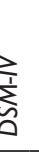 }} &{\text { o }} &{ } &{\sum_{\substack{1 \\
0}}^{\geq}} &{\text {峷 }} &{ } &{ } &{\text { 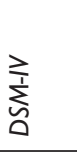 }} \\
{\hline \underline{\infty} \bar{\infty} \hat{\Phi} \hat{m}} &{\text { 三： }} &{6} &{\text { 쏜 } \stackrel{\infty}{\sim}} &{\stackrel{\infty}{=}} &{ } &{ } &{ } &{2 \stackrel{\tilde{\alpha}}{v}} &{ } &{\text { ৪্ল }} &{\text { ర్ల }} &{ } &{8} &{\text { 오 }} \\
{\hline \text { 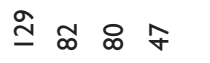 }} &{20} &{\text { ri }} &{\underline{\infty} \stackrel{\infty}{\underline{\omega}}} &{\stackrel{\infty}{\stackrel{\infty}{\varrho}}} &{ } &{ } &{ } &{\stackrel{5}{3}} &{ } &{\stackrel{\infty}{\infty}} &{\text { : }} &{ } &{2} &{\stackrel{\tilde{m}}{\underline{m}}} \\
{\hline \text { 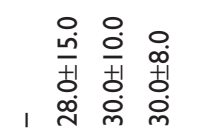 }} &{\begin{array}{l}\hat{0} \\
\dot{0} \\
\vdots \\
0 \\
\infty\end{array}} &{\text { I }} &{\text { 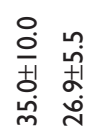 }} &{\text { 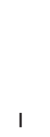 }} &{\text { గ }} &{1} &{\text { I }} &{ } &{ } &{\frac{\stackrel{\infty}{\overline{\overline{+1}}}}{\frac{\dot{m}}{m}}} &{1} &{ } &{\text { 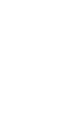 }} &{1} \\
{\hline \text { 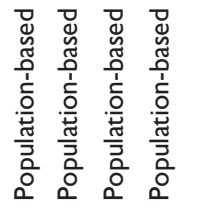 }} &{\text { 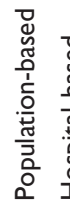 }} &{\text { 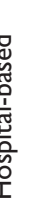 }} &{\text { 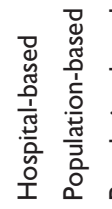 }} &{\text { 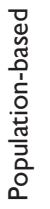 }} &{ } &{ } &{\text { 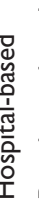 }} &{ } &{ } &{\text { 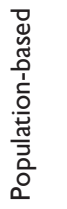 }} &{\text { 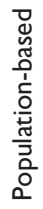 }} &{ } &{\frac{2}{2}} &{\text { 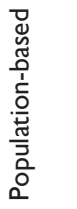 }} \\
{\hline \text { 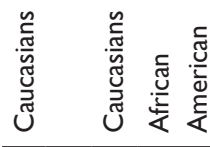 }} &{\text { 秀 }} &{ } &{\text { 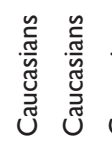 }} &{\text { 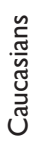 }} &{ } &{ } &{\text { 营 }} &{\text { g }} &{ } &{\text { 胥 }} &{\text { ㄸ్ }} &{ } &{\text { 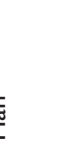 }} &{\text { 疍 }} \\
{\hline \text { 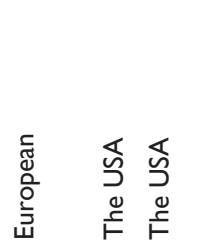 }} &{\text { 离 }} &{ } &{\text { 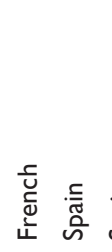 }} &{\text {. } \frac{5}{\pi}} &{ } &{ } &{\text { 苂 }} &{ } &{ } &{\text { 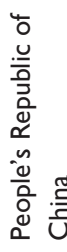 }} &{\text { 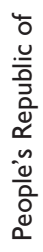 }} &{\text { 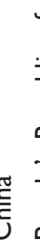 }} &{\text { 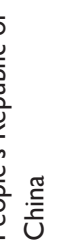 }} &{\text { 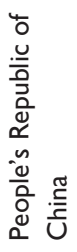 }} \\
{\hline \text { 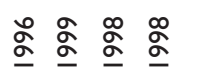 }} &{\overline{\bar{i}} \vdots} &{\text { 20 }} &{\text { 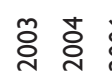 }} &{\text { ঃั }} &{ } &{ } &{\text { 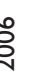 }} &{ } &{ } &{\frac{m}{2}} &{\text { 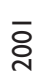 }} &{ } &{\text { 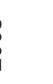 }} &{\frac{\text { ำ }}{2}} \\
{\hline \text { 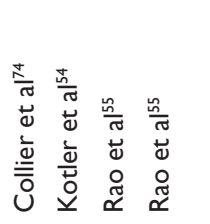 }} &{\text { 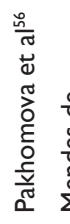 }} &{\begin{array}{ll}0 & \\
0 \\
0\end{array}} &{\text { 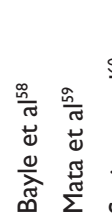 }} &{\text { 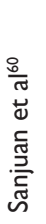 }} &{ } &{ } &{\text { 票 }} &{ } &{ } &{\text { 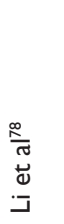 }} &{\text { बु }} &{ } &{ } &{\begin{array}{l}\frac{5}{\pi} \\
\stackrel{0}{0} \\
\frac{0}{20} \\
x\end{array}} \\
$\hline
\end{tabular}


Table 2 Genotype distribution and allele frequency of 5-HTTLPR

\begin{tabular}{|c|c|c|c|c|c|c|c|c|c|c|c|c|}
\hline \multirow[t]{3}{*}{ Authors } & \multirow[t]{3}{*}{ Year } & \multicolumn{6}{|c|}{ Genotype distribution } & \multirow[t]{3}{*}{$P_{\mathrm{HWE}}$} & \multicolumn{4}{|c|}{ Allele frequency } \\
\hline & & \multicolumn{3}{|c|}{ Cases, $\mathrm{n}$} & \multicolumn{3}{|c|}{ Controls, $\mathbf{n}$} & & \multicolumn{2}{|c|}{ Cases, \% } & \multicolumn{2}{|c|}{ Controls, \% } \\
\hline & & LL & LS & SS & LL & LS & SS & & $\mathbf{L}$ & $\mathbf{S}$ & $\mathbf{L}$ & $\mathbf{S}$ \\
\hline Pae et $\mathrm{al}^{36}$ & 2005 & 4 & 35 & 72 & 10 & 49 & 113 & 0.143 & 43 & 179 & 69 & 275 \\
\hline Saiz & 2007 & 64 & 104 & 59 & 124 & 203 & 93 & 0.566 & 232 & 222 & 451 & 389 \\
\hline Naylor et $\mathrm{al}^{38}$ & 1998 & 11 & 30 & 17 & 19 & 29 & 14 & 0.646 & 52 & 64 & 67 & 57 \\
\hline Ikeda et al $\left.\right|^{39}$ & 2006 & 16 & 82 & 189 & 12 & 101 & 175 & 0.588 & 114 & 460 & 125 & 451 \\
\hline Vijayan et al ${ }^{40}$ & 2009 & 40 & 123 & 79 & 62 & 118 & 61 & 0.748 & 203 & 281 & 242 & 240 \\
\hline Lee et $\mathrm{a}^{41}$ & 2009 & 7 & 52 & 82 & 3 & 45 & 67 & 0.151 & 66 & 216 & 51 & 179 \\
\hline Pae et $\mathrm{al}^{42}$ & 2003 & 4 & 35 & 72 & 11 & 58 & 139 & 0.140 & 43 & 179 & 80 & 336 \\
\hline Gu et $\mathrm{a}^{43}$ & 2013 & 14 & 101 & 289 & 7 & 90 & 288 & 0.992 & 129 & 679 & 104 & 666 \\
\hline Kaiser et al ${ }^{16}$ & 2001 & 253 & 324 & 107 & 207 & 286 & 94 & 0.772 & 830 & 538 & 700 & 474 \\
\hline Herken et al ${ }^{44}$ & 2003 & 30 & 70 & 40 & 17 & 24 & 27 & 0.022 & 130 & 150 & 58 & 78 \\
\hline Serretti et a $\left.\right|^{45}$ & 2002 & 89 & $12 \mid$ & 51 & 165 & 217 & 75 & 0.800 & 299 & 223 & 547 & 367 \\
\hline Tsai et $a^{67}$ & 1999 & 9 & 28 & 53 & 15 & 30 & 59 & 0.002 & 46 & 134 & 60 & 148 \\
\hline Han et al ${ }^{46}$ & 2004 & 7 & 52 & 109 & 8 & 54 & 96 & 0.910 & 66 & 270 & 70 & 246 \\
\hline Zaboli et a ${ }^{47}$ & 2008 & 56 & 73 & 26 & 75 & 126 & 45 & 0.532 & 185 & 125 & 276 & 216 \\
\hline Golimbet et $\mathrm{a}^{48}$ & 2017 & 486 & 595 & 204 & 413 & 503 & 145 & 0.677 & 1,567 & 1,003 & 1,329 & 793 \\
\hline Peitl et al ${ }^{49}$ & 2017 & 118 & 124 & 58 & 103 & 138 & 50 & 0.746 & 360 & 240 & 344 & 238 \\
\hline Terzic & 2015 & 52 & 71 & 15 & 47 & 34 & 13 & 0.104 & 175 & 101 & 128 & 60 \\
\hline Lu et $\mathrm{a}^{68}$ & 2012 & 407 & 626 & 254 & 690 & 992 & 345 & 0.719 & $\mathrm{I}, 440$ & $\mathrm{I}, 134$ & 2,372 & 1,682 \\
\hline Golimbet et $\mathrm{a}^{51}$ & 2010 & 27 & 20 & 11 & 22 & 28 & 12 & 0.567 & 74 & 42 & 72 & 52 \\
\hline Golimbet et $\mathrm{al}^{52}$ & 2003 & 42 & 46 & 22 & 45 & 57 & 22 & 0.594 & 130 & 90 & 147 & 101 \\
\hline Stober & 1998 & 58 & 90 & 32 & 79 & 112 & 42 & 0.834 & 206 & 154 & 270 & 196 \\
\hline Kotler et $\mathrm{a}^{54}$ & 1999 & 24 & 38 & 20 & 210 & 446 & 165 & 0.010 & 86 & 78 & 866 & 776 \\
\hline Rao et $\mathrm{al}^{55}$ & 1998 & 28 & 32 & 20 & 43 & 59 & 25 & 0.559 & 88 & 72 & 145 & 109 \\
\hline Rao et $\mathrm{al}^{55}$ & 1998 & 25 & 18 & 4 & 18 & 12 & 7 & 0.079 & 68 & 26 & 48 & 26 \\
\hline Pakhomova & 2011 & 20 & 37 & 8 & 43 & 48 & 20 & 0.310 & 77 & 53 & 134 & 88 \\
\hline Mendes de & 1998 & 12 & 21 & 6 & 39 & 43 & 16 & 0.480 & 45 & 33 & 121 & 75 \\
\hline Oliveira et $\mathrm{al}^{57}$ & & & & & & & & & & & & \\
\hline Bayle et al ${ }^{58}$ & 2003 & 48 & 99 & 38 & 40 & 83 & 36 & 0.573 & 195 & 175 & 163 & 155 \\
\hline Mata et al ${ }^{59}$ & 2004 & $4 I$ & 102 & 44 & 63 & 124 & 91 & 0.100 & 184 & 190 & 250 & 306 \\
\hline Sanjuan et a $\left.\right|^{60}$ & 2006 & 33 & 78 & 47 & 44 & 68 & 26 & 0.976 & 144 & 172 & 156 & 120 \\
\hline Chong et $\mathrm{al}^{61}$ & 2000 & 33 & 130 & 175 & 13 & 32 & 58 & 0.019 & 196 & 480 & 58 & 148 \\
\hline Pae et $a^{62}$ & 2006 & 5 & 46 & 101 & 9 & 47 & 96 & 0.322 & 56 & 248 & 65 & 239 \\
\hline Young & 2006 & 2 & 5 & 5 & 6 & 7 & 2 & 0.985 & 9 & 15 & 19 & 11 \\
\hline Jing et $\mathrm{a}^{64}$ & 2016 & 51 & 267 & 306 & 66 & 248 & 369 & 0.012 & 369 & 879 & 380 & 986 \\
\hline Li et $\mathrm{al}^{78}$ & 2013 & 5 & 35 & 149 & 24 & 134 & 142 & 0.325 & 45 & 333 & 182 & 418 \\
\hline Zuo & 2003 & 15 & 50 & 92 & 21 & 49 & 115 & 0.000 & 80 & 234 & 91 & 279 \\
\hline
\end{tabular}

Abbreviations: 5-HTTLPR, serotonin-transporter-linked polymorphic region; $P_{\text {HWE }}{ }^{\prime}$-value of Hardy-Weinberg equilibrium.

risk factor for schizophrenia $\left(P_{z}=0.006, \mathrm{OR}=1.299,95 \%\right.$ $\mathrm{CI}=1.079-1.565)$, with a power of 0.893 . No significant heterogeneity was observed in the pooled or subgroup analyses.

\section{No association between rs 140700 and the risk of schizophrenia}

Under a random model, the allele frequencies of 1,770 cases and 2,386 controls were included in the pooled and subgroup analyses (Table 7). In a dominant model, no association was detected between rs140700 and the risk of schizophrenia in the pooled and subgroup analyses (Tables 3 and 4). Significant heterogeneity was observed in the pooled
$\left(P_{h}=0.000, I^{2}=93.2 \%\right)$ and East Asia subgroup $\left(P_{h}=0.000\right.$, $I^{2}=93.5 \%$ ) analyses.

\section{Sensitivity analysis}

We conducted sensitivity analyses by omitting each study individually; the pooled ORs did not change significantly. Thus, the results were considered stable and reasonable.

\section{Publication bias}

Any publication bias was made visible by funnel plots, in which the SE of the log OR of each study was plotted against its $\log$ OR. No evidence of publication bias was found in the pooled analyses (Figures 2-7). 
Table 3 Pooled association of SLC6A4 polymorphisms with schizophrenia

\begin{tabular}{|c|c|c|c|c|c|c|c|c|c|}
\hline Loci & Genetic model & Studies (n) & Statistical & OR & $95 \% \mathrm{Cl}$ & $P_{z}$ & $I^{2}$ & $\boldsymbol{P}_{h}$ & $P_{\mathrm{e}}$ \\
\hline \multirow[t]{5}{*}{ 5-HTTLPR } & Allele contrast & 35 & Random & 0.934 & $0.868-1.004$ & 0.066 & 53.6 & 0.000 & 0.587 \\
\hline & Homozygous codominant & 35 & Random & 1.142 & $0.782-0.980$ & 0.021 & 15.6 & 0.206 & 0.912 \\
\hline & Heterozygous codominant & 35 & Random & 1.066 & $0.980-1.161$ & 0.138 & 7.7 & 0.340 & 0.165 \\
\hline & Dominant & 35 & Random & 1.085 & $0.999-1.178$ & 0.054 & 10.4 & 0.294 & 0.211 \\
\hline & Recessive & 35 & Random & 1.082 & $0.96 \mathrm{I}-1.218$ & 0.195 & 57.8 & 0.000 & 0.846 \\
\hline \multirow[t]{5}{*}{ STin2 VNTR } & IOR vs others & 17 & Random & 0.884 & $0.725-1.077$ & 0.221 & 82.8 & 0.000 & 0.951 \\
\hline & I $2 \mathrm{R}$ vs others & 17 & Random & 1.134 & $0.93|-| .38 \mid$ & 0.21 & 82.8 & 0.000 & 0.936 \\
\hline & IOR/IOR vs others & 17 & Random & 0.907 & $0.624-1.319$ & 0.611 & 78.9 & 0.000 & 0.401 \\
\hline & $10 R / I 2 R$ vs others & 17 & Random & 0.789 & $0.646-0.963$ & 0.020 & 71.1 & 0.000 & 0.748 \\
\hline & $12 R / I 2 R$ vs others & 17 & Random & 1.936 & $1.238-3.029$ & 0.004 & 94.7 & 0.000 & 0.474 \\
\hline \multirow[t]{5}{*}{ rs 1042173} & Contrast & 4 & Random & 1.094 & $0.962-1.245$ & 0.17 & 22.7 & 0.275 & 0.412 \\
\hline & Homozygous codominant & 4 & Random & 1.214 & $0.921-1.600$ & 0.169 & 26.4 & 0.253 & 0.336 \\
\hline & Heterozygous codominant & 4 & Random & 1.011 & $0.812-1.260$ & 0.920 & 18.0 & 0.301 & 0.941 \\
\hline & Dominant & 4 & Random & 1.077 & $0.856-1.354$ & 0.528 & 26.9 & 0.250 & 0.722 \\
\hline & Recessive & 4 & Random & 1.199 & $0.994-1.445$ & 0.057 & 14.5 & 0.320 & 0.155 \\
\hline \multirow[t]{5}{*}{ rs 140700} & Allele contrast & 4 & Random & 1.052 & $0.519-1.734$ & 0.863 & 92.9 & 0.000 & 0.359 \\
\hline & Homozygous codominant & 4 & Random & 0.809 & $0.236-2.774$ & 0.736 & 63.7 & 0.041 & 0.223 \\
\hline & Heterozygous codominant & 4 & Random & 0.971 & $0.500-1.884$ & 0.931 & 91.9 & 0.000 & 0.685 \\
\hline & Dominant & 4 & Random & 1.003 & $0.503-1.999$ & 0.993 & 93.2 & 0.000 & 0.560 \\
\hline & Recessive & 4 & Random & 0.830 & $0.284-2.422$ & 0.733 & 53.5 & 0.092 & 0.333 \\
\hline
\end{tabular}

Abbreviations: 5-HTTLPR, serotonin-transporter-linked polymorphic region; $P_{z}, P_{h}$, and $P_{\mathrm{e}}, P$-values of association, heterogeneity, and publication bias tests, respectively; $R$, repeats; STin2 VNTR, second intron variable number of tandem repeats.

Table 4 Subgroup association of SLC6A4 polymorphisms with schizophrenia

\begin{tabular}{|c|c|c|c|c|c|c|c|}
\hline Loci & Subgroup analysis & Studies (n) & OR & $95 \% \mathrm{Cl}$ & $P_{z}$ & $1^{2}$ & $\boldsymbol{P}_{h}$ \\
\hline \multirow[t]{6}{*}{5 HTTLPR } & Caucasians & 21 & 1.053 & $0.974-1.138$ & 0.192 & 1.6 & 0.437 \\
\hline & East Asia & 12 & 1.204 & $0.953-1.52 \mid$ & 0.119 & 4.1 & 0.404 \\
\hline & African American & 1 & 0.834 & $0.352-1.975$ & 0.679 & 0.0 & 0.000 \\
\hline & Indians & I & 1.749 & $|| 20-2.73 \mid$. & 0.014 & 0.0 & 0.000 \\
\hline & Population-based & 26 & 1.089 & $0.982-1.209$ & 0.131 & 28.3 & 0.090 \\
\hline & Hospital-based & 9 & 1.094 & $0.897-1.335$ & 0.273 & 0.0 & 0.939 \\
\hline \multirow[t]{5}{*}{ STin2 VNTR 1010} & Caucasians & 9 & 0.883 & $0.645-1.207$ & 0.435 & 63.7 & 0.005 \\
\hline & East Asia & 7 & 0.857 & $0.310-2.374$ & 0.767 & 75.5 & 0.00 \\
\hline & Indians & I & 0.639 & $0.368-1.108$ & 0.111 & - & 0.000 \\
\hline & Population-based & 16 & 0.930 & $0.639-1.354$ & 0.704 & 79.8 & 0.000 \\
\hline & Hospital-based & 1 & 0.155 & $0.008-3.040$ & 0.220 & - & - \\
\hline \multirow[t]{5}{*}{1012} & Caucasians & 9 & 0.944 & $0.832-1.072$ & 0.374 & 0.0 & 0.706 \\
\hline & East Asia & 7 & 0.617 & $0.389-0.978$ & 0.040 & 80.8 & 0.000 \\
\hline & Indians & I & 0.635 & $0.44 I-0.913$ & 0.014 & - & - \\
\hline & Population-based & 16 & 0.794 & $0.646-0.976$ & 0.028 & 72.8 & 0.000 \\
\hline & Hospital-based & 1 & 0.667 & $0.290-1.536$ & 0.341 & - & - \\
\hline \multirow[t]{5}{*}{1212} & Caucasians & 9 & 1.042 & $0.916-1.186$ & 0.531 & 0.0 & 0.885 \\
\hline & East Asia & 7 & 4.482 & $2.312-8.689$ & 0.000 & 92.1 & 0.000 \\
\hline & Indians & 1 & 1.573 & $1.086-2.280$ & 0.170 & - & - \\
\hline & Population-based & 16 & 1.755 & I.124-2.742 & 0.013 & 94.5 & 0.000 \\
\hline & Hospital-based & I & 10.689 & $5.303-21.544$ & 0.000 & - & - \\
\hline \multirow[t]{3}{*}{ rs1042173 } & Caucasians & 2 & 1.299 & I.0791.565 & 0.006 & 0.0 & 0.421 \\
\hline & East Asia & 1 & 0.834 & 0.5171 .346 & 0.458 & - & - \\
\hline & Indians & I & 1.213 & 0.7981 .843 & 0.365 & - & - \\
\hline \multirow[t]{2}{*}{ rs 140700} & Caucasians & 2 & 1.253 & 0.8271 .896 & 0.287 & 42.6 & 0.187 \\
\hline & East Asia & 2 & 0.757 & 0.2152 .670 & 0.665 & 93.5 & 0.000 \\
\hline
\end{tabular}

Abbreviations: 5-HTTLPR, serotonin-transporter-linked polymorphic region; $P_{z}$ and $P_{h}, P$-values of association and heterogeneity, respectively; STin2 VNTR, second intron variable number of tandem repeats. 
Table 5 Genotype distribution and allele frequency of STin2 VNTR

\begin{tabular}{|c|c|c|c|c|c|c|c|c|c|c|c|c|c|c|c|}
\hline \multirow[t]{3}{*}{ Authors } & \multirow[t]{3}{*}{ Year } & \multicolumn{6}{|c|}{ Genotype distribution } & \multicolumn{8}{|c|}{ Allele frequency } \\
\hline & & \multicolumn{3}{|c|}{ Case } & \multicolumn{3}{|c|}{ Control } & \multicolumn{4}{|l|}{ Case } & \multirow[b]{2}{*}{1010} & \multicolumn{3}{|c|}{ Control } \\
\hline & & 10 & 12 & Others & 10 & 12 & Others & 1010 & 1012 & 1212 & Others & & 1012 & 1212 & Others \\
\hline Frtdtrique & 1997 & 84 & 120 & 6 & 79 & 143 & 6 & 24 & 36 & 39 & 6 & 18 & 41 & 50 & 5 \\
\hline Saiz & 2007 & 171 & 281 & 2 & 305 & 530 & 5 & 42 & 86 & 97 & 2 & 64 & 177 & 174 & 5 \\
\hline Liu et $\mathrm{al}^{70}$ & 1999 & 22 & 498 & 0 & 58 & 665 & 1 & 2 & 18 & 240 & 0 & 5 & 48 & 308 & I \\
\hline Ikeda et $\mathrm{al}^{39}$ & 2006 & 51 & 523 & 0 & 46 & 530 & 0 & I & 49 & 237 & 0 & 3 & 40 & 245 & 0 \\
\hline Lin et $\mathrm{al}^{71}$ & 2009 & 55 & 425 & 0 & 107 & 457 & 0 & 6 & 43 & 191 & 0 & 9 & 89 & 184 & 0 \\
\hline Vijayan et a ${ }^{40}$ & 2009 & 137 & 343 & 0 & 189 & 297 & 0 & 24 & 89 & 127 & 0 & 36 & 117 & 90 & 0 \\
\hline Lee et $\mathrm{al}^{4 l}$ & 2009 & 36 & 246 & 0 & 23 & 207 & 0 & 4 & 28 & 109 & 0 & 3 & 17 & 95 & 0 \\
\hline Kaiser et al ${ }^{16}$ & 2001 & 493 & 835 & 40 & 485 & 668 & 21 & 95 & 291 & 259 & 39 & 108 & 259 & 199 & 21 \\
\hline Tsai et $\mathrm{al}^{72}$ & 2002 & 10 & 218 & 0 & 22 & 232 & 0 & 0 & 10 & 104 & 0 & 3 & 16 & 108 & 0 \\
\hline Herken et $\mathrm{a}^{44}$ & 2003 & 82 & 204 & 0 & 31 & 59 & 0 & 12 & 58 & 73 & 0 & 8 & 15 & 22 & 0 \\
\hline Zaboli et al ${ }^{47}$ & 2008 & 122 & 184 & 0 & 176 & 302 & 0 & 30 & 62 & 61 & 0 & 29 & 118 & 92 & 0 \\
\hline Herken et $\mathrm{a}^{73}$ & 2002 & 72 & 184 & 0 & 71 & 198 & 1 & 9 & 54 & 65 & 0 & 7 & 57 & 70 & I \\
\hline Stober & 1998 & 141 & 217 & 2 & 204 & 254 & 8 & 29 & 83 & 66 & 2 & 54 & 96 & 75 & 8 \\
\hline Collier et $\mathrm{al}^{74}$ & 1996 & 94 & 162 & 2 & 169 & 202 & 3 & 19 & 56 & 52 & 2 & 42 & 85 & 57 & 3 \\
\hline Mata et al ${ }^{59}$ & 2004 & 103 & 221 & 0 & 178 & 288 & 0 & 15 & 73 & 74 & 0 & 37 & 104 & 92 & 0 \\
\hline Li et $\mathrm{al}^{78}$ & 2013 & 333 & 45 & 0 & 418 & 182 & 0 & 149 & 35 & 5 & 0 & 142 & 134 & 24 & 0 \\
\hline Yang & 2001 & 22 & 498 & 0 & 58 & 665 & 1 & 2 & 18 & 240 & 0 & 5 & 48 & 308 & I \\
\hline
\end{tabular}

Abbreviation: STin2 VNTR, second intron variable number of tandem repeats.

\section{Discussion}

We found no association between 5-HTTLPR and the risk of schizophrenia, except in Indians. The scale for Indians was small and was found in only one article; therefore, the association may be a false-positive. A negative association between 5-HTTLPR and the risk of schizophrenia was consistent with the results of the previous meta-analyses, ${ }^{19,21}$ but inconsistent with the results of Allen et al..$^{22}$ Differences may exist in results found because Allen et al only analyzed articles on the SzGene in their meta-analysis. An association between suicidal behavior and 5-HTTLPR was not detected in a recent meta-analysis, ${ }^{83}$ which conflicts with previous evidence suggesting an association between 5-HTTLPR and violent suicidal behavior. The $\mathrm{L}$ allele of the 5-HTTLPR was reported as improving transcription of the SLC6A4 gene. ${ }^{84}$ A meta-analysis noted an association between the $\mathrm{S}$ allele of 5-HTTLPR and the risk of bipolar disorder ${ }^{85}$ Psychiatric disorders share genetic variants. ${ }^{21}$ A haplotype, including
5-HTTLPR and rs16965628 markers, is thought to be associated with an obsessive-compulsive disorder ${ }^{86}$ Therefore, 5-HTTLPR may link with other SNPs to influence the serotoninergic pathway.

STin2 VNTR was associated with the risk of schizophrenia, but a significant difference was not detected in the allele analysis, inconsistent with other meta-analyses. ${ }^{19,20}$ Gatt et al reviewed the relevant meta-analysis between STin2 VNTR and schizophrenia and found that the 12R genotype was associated with schizophrenia as a protective factor, while $9 R$ and 10R genotypes were not associated with schizophrenia. ${ }^{21}$ Our results showed that the $10 \mathrm{R} / 12 \mathrm{R}$ genotype was a protective factor for schizophrenia, while the $12 \mathrm{R} / 12 \mathrm{R}$ genotype was a risk factor for schizophrenia, in the pooled and several subgroup analyses. Genotypes with $12 \mathrm{R}$ may significantly increase relative 5-HTT gene expression, ${ }^{87}$ leading to increasing vulnerability to schizophrenia. STin2.12R has a superior enhancerlike property within the developing rostral hindbrain, which

Table 6 Genotype distribution and allele frequency of rs 1042173

\begin{tabular}{|c|c|c|c|c|c|c|c|c|c|c|c|c|}
\hline \multirow[t]{3}{*}{ Authors } & \multirow[t]{3}{*}{ Year } & \multicolumn{6}{|c|}{ Genotype distribution } & \multirow[t]{3}{*}{$P_{\text {HWE }}$} & \multicolumn{4}{|c|}{ Allele frequency } \\
\hline & & \multicolumn{3}{|c|}{ Cases, $\mathbf{n}$} & \multicolumn{3}{|c|}{ Controls, $n$} & & \multicolumn{2}{|c|}{ Cases, \% } & \multicolumn{2}{|c|}{ Controls, $\%$} \\
\hline & & GG & GT & TT & GG & GT & TT & & G & $\mathbf{T}$ & G & $\mathbf{T}$ \\
\hline Vijayan et al ${ }^{40}$ & 2009 & 63 & 119 & 50 & 55 & 112 & 67 & 0.538 & 245 & 219 & 222 & 246 \\
\hline Zaboli et $\mathrm{al}^{47}$ & 2008 & 39 & 78 & 38 & 58 & 129 & 59 & 0.444 & 156 & 154 & 245 & 247 \\
\hline Carlstrom & 2012 & 207 & 402 & 223 & 291 & 770 & 410 & 0.039 & 816 & 848 & 1,352 & 1,590 \\
\hline Xuan et $\mathrm{al}^{77}$ & 2012 & 77 & 43 & 12 & 94 & 48 & 8 & 0.568 & 197 & 67 & 236 & 64 \\
\hline
\end{tabular}

Abbreviation: $P_{\mathrm{HWE}}, P$-value of Hardy-Weinberg equilibrium. 
Table 7 Genotype distribution and allele frequency of rs 140700

\begin{tabular}{|c|c|c|c|c|c|c|c|c|c|c|c|c|}
\hline \multirow[t]{3}{*}{ Authors } & \multirow[t]{3}{*}{ Year } & \multicolumn{6}{|c|}{ Genotype distribution } & \multirow[t]{3}{*}{$P_{\text {HWE }}$} & \multicolumn{4}{|c|}{ Allele frequency } \\
\hline & & \multicolumn{3}{|c|}{ Cases, $n$} & \multicolumn{3}{|c|}{ Controls, $\mathbf{n}$} & & \multicolumn{2}{|c|}{ Cases, \% } & \multicolumn{2}{|c|}{ Controls, \% } \\
\hline & & GG & GA & AA & GG & GA & AA & & G & $\mathbf{A}$ & $\mathbf{G}$ & $\mathbf{A}$ \\
\hline Li et $\mathrm{al}^{78}$ & 2013 & 369 & 127 & 30 & 259 & 212 & 57 & 0.173 & 865 & 187 & 730 & 326 \\
\hline Lin et $\mathrm{al}^{71}$ & 2009 & 277 & 30 & 2 & 244 & 18 & 1 & 0.295 & 584 & 34 & 506 & 20 \\
\hline Carlstrom & 2012 & 689 & 140 & 2 & 1,233 & 221 & 10 & 0.977 & 1,518 & 144 & 2,687 & $24 I$ \\
\hline Pal et $\mathrm{al}^{79}$ & 2009 & 80 & 19 & 5 & 112 & 18 & 1 & 0.769 & 179 & 29 & 242 & 20 \\
\hline
\end{tabular}

Abbreviation: $P_{\text {HWE' }}, P$-value of Hardy-Weinberg equilibrium.

may lead to aberrant serotonergic neuronal development. ${ }^{40,88}$ In addition, STin2 acts as a transcriptional regulator in an allele-dependent manner in the developing mouse brain. ${ }^{89}$ Haplotype analysis demonstrated that two STin2-containing haplotypes were associated with the risk of schizophrenia, but no association was found in the single locus. ${ }^{71}$ No association was detected in Caucasians and Indians, which may be the result of different genetic backgrounds. Significant heterogeneity was assessed in the pooled analysis, and heterogeneity was found in all subgroups, except Caucasians. This was the first meta-analysis of the association between the risk of schizophrenia and rs1042173 and rs 140700 .

Gatt et al comprehensively reviewed a meta-analysis of the association between SLC6A4 (5-HTTLPR and STin2 VNTR) and schizophrenia. ${ }^{21}$ To some extent, it would seem that our meta-analysis is superfluous. However, it was an updated analysis, assessing the association of the $S L C 6 A 4$ gene with schizophrenia using high statistical powers. Our study also included seven studies published after 2013 and three studies in Chinese. Moreover, four variations (5-HTTLPR, STin2 VNTR, rs1042173, and rs140700) were analyzed in our

\section{Funnel plot with pseudo $95 \% \mathrm{Cl}$}

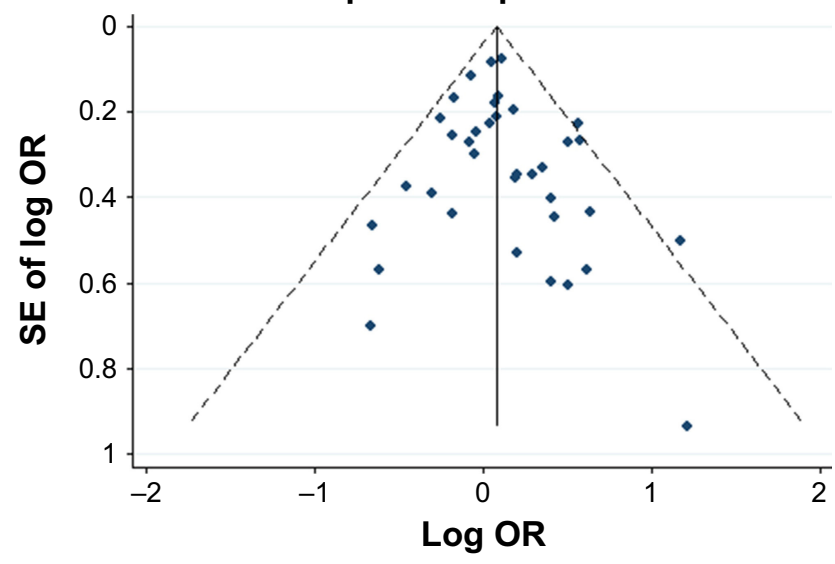

Figure 2 Funnel plot analysis on the detection of publication bias in the association between 5-HTTLPR (SS + LS vs LL) and schizophrenia.

Abbreviation: 5-HTTLPR, serotonin-transporter-linked polymorphic region. meta-analysis. Genome-wide association studies (GWASs) can discover novel and unexpected candidate loci in an unbiased manner. Previous GWAS analyses found that the SLC6A4 gene was not associated with schizophrenia. ${ }^{90-93}$ A comparison of 12 single-disorder GWAS meta-analyses suggested no overlap in significant genetic variants identified from the different studies. ${ }^{21}$ However, structural magnetic resonance imaging scans suggested that $S L C 6 A 4$ was related to deficits of brain structural networks in schizophrenia. ${ }^{94}$ Our results are inconsistent with those of the previous meta-analysis. Several reasons for this may exist: First, many recently published studies were included in our analysis; therefore, the scale of samples used was larger than those used before. Second, the articles from both English and Chinese language databases were included. Third, geographical environment, culture, lifestyle, and genetic background and diseases may affect genetic polymorphisms. ${ }^{95,96}$

Significant heterogeneity was found in overall and subgroup analyses, especially for STin2 VNTR and rs140700. Although we performed subgroup analyses according to ethnicity to investigate potential sources of heterogeneity,

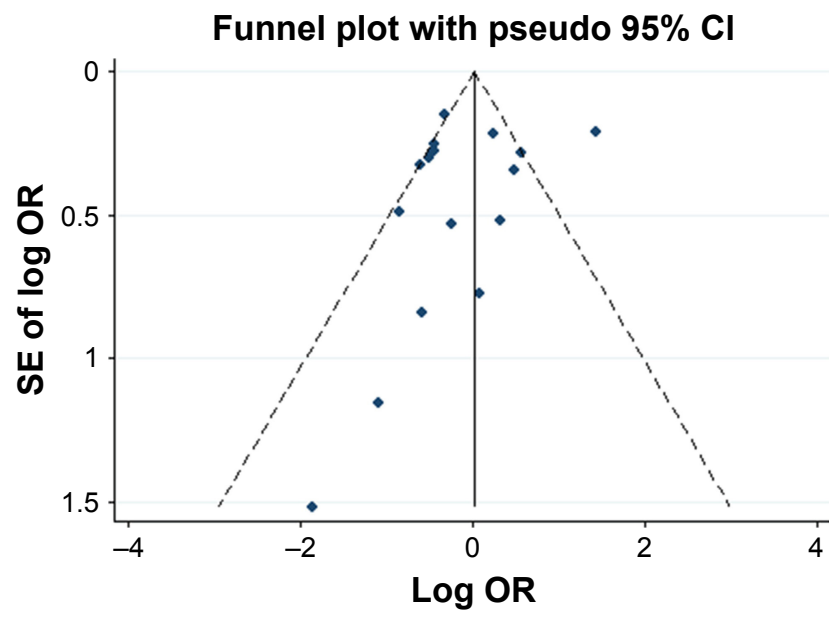

Figure 3 Funnel plot analysis on the detection of publication bias in the association between STin2 VNTR (IOR/IOR vs others) and schizophrenia.

Abbreviation: STin2 VNTR, second intron variable number of tandem repeats. 


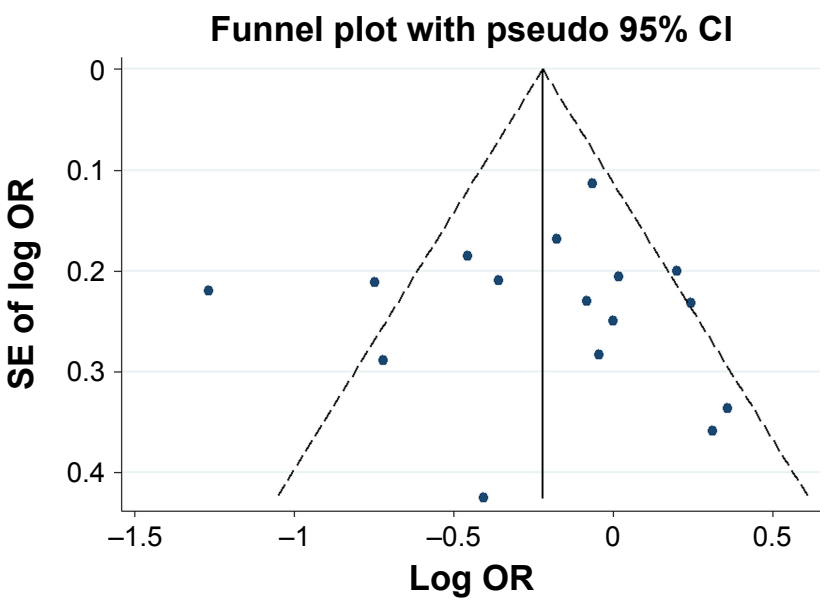

Figure 4 Funnel plot analysis on the detection of publication bias in the association between STin2 VNTR (IOR/I2R vs others) and schizophrenia.

Abbreviation: STin2 VNTR, second intron variable number of tandem repeats.

this did not completely account for the heterogeneity. These results suggest that other aspects may partially contribute to heterogeneity, such as distinct genetic backgrounds and the different habits and customs of the people sampled. ${ }^{97}$

Overall, however, the results described herein should be interpreted with caution. First, the small sample size for rs1042173 and rs140700 should be borne in mind. Several associations only appeared in the subgroup analyses, for which only one or two articles were used. Therefore, these samples may not be representative and comprehensive. In addition, it was hard to conduct subgroup analyses for some SNPs because of so few articles. Second, deviations in the $P_{\text {HWE }}$ and significant heterogeneity were observed in this study because of sample bias. Third, family-based studies, which were more robust than case-control designs, were not included in this analysis. ${ }^{98-101}$ Fourth, interactions between

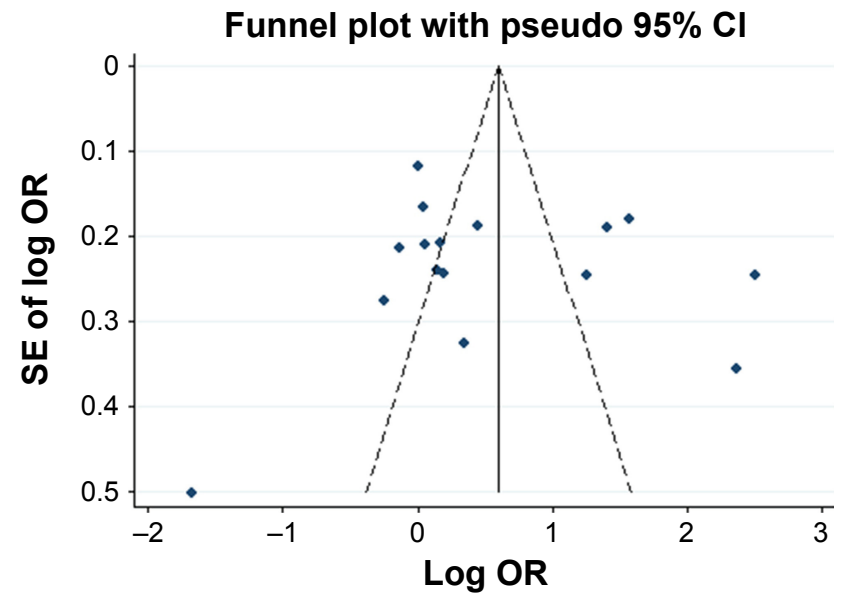

Figure 5 Funnel plot analysis on the detection of publication bias in the association between STin2 VNTR (I2R/I2R vs others) and schizophrenia.

Abbreviation: STin2 VNTR, second intron variable number of tandem repeats.

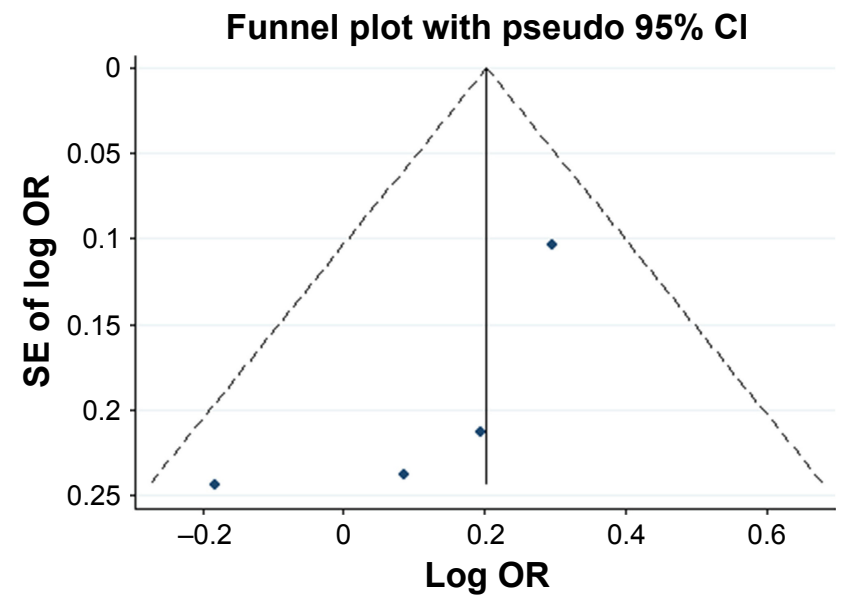

Figure 6 Funnel plot analysis on the detection of publication bias in the association between rs 1042173 (GG vs GT + TT) and schizophrenia.

multiple genes and SNPs may affect the risk of schizophrenia, ${ }^{21}$ meaning that genetic interactional and functional studies are needed.

\section{Conclusion}

Our meta-analysis showed a lack of association between 5-HTTLPR and the risk of schizophrenia, except in an Indian subgroup analysis. The $10 \mathrm{R} / 12 \mathrm{R}$ genotype was a protective factor against schizophrenia, while the $12 \mathrm{R} / 12 \mathrm{R}$ genotype was a risk factor for schizophrenia in the pooled analyses. In Caucasians, the GG genotype of rs 1042173 may be a risk factor for schizophrenia. No association was found between rs140700 and the risk of schizophrenia. Increased genetic interactional and functional studies are warranted to explore the association between polymorphisms of the SLC6A4 gene and schizophrenia risk.

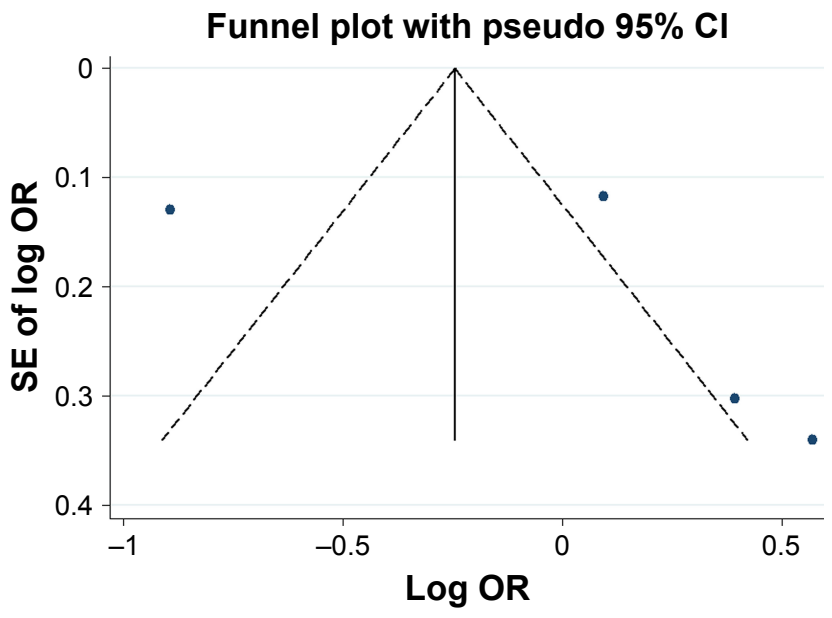

Figure 7 Funnel plot analysis on the detection of publication bias in the association between rs 140700 (AA + AG vs GG) and schizophrenia. 


\section{Disclosure}

The authors report no conflicts of interest in this work.

\section{References}

1. Wray NR, Visscher PM. Narrowing the boundaries of the genetic architecture of schizophrenia. Schizophr Bull. 2010;36(1):14-23.

2. Sullivan PF. The genetics of schizophrenia. PLoS Med. 2005;2(7):e212.

3. Uher R. Gene-environment interactions in severe mental illness. Front Psychiatry. 2014;5(9):48.

4. Cardno AG, Gottesman II. Twin studies of schizophrenia: from bowand-arrow concordances to star wars $\mathrm{Mx}$ and functional genomics. $\mathrm{Am}$ J Med Genet. 2000;97(1):12-17.

5. Ohno Y. New insight into the therapeutic role of 5-HT1A receptors in central nervous system disorders. Cent Nerv Syst Agents Med Chem. 2010;10(2):148-157.

6. Mohammadi A, Rashidi E, Amooeian VG. Brain, blood, cerebrospinal fluid, and serum biomarkers in schizophrenia. Psychiatry Res. 2018;265:25-38

7. Kim JH, Kim JH, Son YD, et al. Altered interregional correlations between serotonin transporter availability and cerebral glucose metabolism in schizophrenia: A high-resolution PET study using [(11)C]DASB and [(18)F]FDG. Schizophr Res. 2017;182:55-65.

8. Watanabe SY, Numata S, Iga JI, et al. Gene expression-based biological test for major depressive disorder: an advanced study. Neuropsychiatr Dis Treat. 2017;13:535-541.

9. Brusov OS, Faktor MI, Zlobina GP, et al. Urovni i molekulyarnyye geterogennost' belka-perenoschika serotonina $\mathrm{v}$ trombotsitakh patsiyentov s razlichnymi psikhicheskimi zabolevaniyami: sravnitel'nyy analiz s ispol'zovaniyem monoklonal'nyye i poliklonal'nyye antitela. [Levels and molecular heterogeneity of serotonin transporter protein in platelets of patients with different mental diseases: a comparative analysis with the use of monoclonal and polyclonal antibodies]. Vestn Ross Akad Med Nauk. 2001;7:37-42. Russian.

10. Gelernter J, Pakstis AJ, Kidd KK. Linkage mapping of serotonin transporter protein gene SLC6A4 on chromosome 17. Hum Genet. 1995; 95(6):677-680.

11. Health Quality Ontario. Pharmacogenomic testing for psychotropic medication selection: a systematic review of the Assurex GeneSight Psychotropic Test. Ont Health Technol Assess Ser. 2017;17(4):1-39.

12. Bilic P, Jukic V, Vilibic M, Savic A, Bozina N. Treatment-resistant schizophrenia and DAT and SERT polymorphisms. Gene. 2014;543(1): $125-132$.

13. Terzić T, Kastelic M, Dolžan V, Plesničar BK. Influence of 5-HT1A and 5-HTTLPR genetic variants on the schizophrenia symptoms and occurrence of treatment-resistant schizophrenia. Neuropsychiatr Dis Treat. 2015;11:453-459.

14. Tsai SJ, Hong CJ, Liou YJ. Recent molecular genetic studies and methodological issues in suicide research. Prog Neuropsychopharmacol Biol Psychiatry. 2011;35(4):809-817.

15. Yang B, Huang X, Ruan L, et al. No association of SLC6A3 and SLC6A4 gene polymorphisms with schizophrenia in the Han Chinese population. Neurosc Lett. 2014;579:114-118.

16. Kaiser R, Tremblay P-B, Schmider J, et al. Serotonin transporter polymorphisms: no association with response to antipsychotic treatment, but associations with the schizoparanoid and residual subtypes of schizophrenia. Molecular Psychiatry. 2001;6(2):179-185.

17. Kebede MM, Zeeb H, Peters M, Heise TL, Pischke CR. Effectiveness of Digital Interventions for Improving Glycemic Control in Persons with Poorly Controlled Type 2 Diabetes: A Systematic Review, Metaanalysis, and Meta-regression Analysis. Diabetes Technol Ther. 2018; 20(11):767-782.

18. Lee J. The Relationship Between Physical Activity and Dementia: A Systematic Review and Meta-Analysis of Prospective Cohort Studies. J Gerontol Nurs. 2018;44(10):22-29.

19. Fan JB, Sklar P. Meta-analysis reveals association between serotonin transporter gene STin2 VNTR polymorphism and schizophrenia. Mol Psychiatry. 2005;10(10):928-938.
20. Shi J, Gershon ES, Liu C. Genetic associations with schizophrenia: meta-analyses of 12 candidate genes. Schizophr Res. 2008;104(1-3): 96-107.

21. Gatt JM, Burton KL, Williams LM, Schofield PR. Specific and common genes implicated across major mental disorders: a review of metaanalysis studies. $J$ Psychiatr Res. 2015;60:1-13.

22. Allen NC, Bagade S, McQueen MB, et al. Systematic meta-analyses and field synopsis of genetic association studies in schizophrenia: the SzGene database. Nat Genet. 2008;40(7):827-834.

23. Munafò MR, Flint J. Meta-analysis of genetic association studies. Trends Genet. 2004;20(9):439-444.

24. Myung SK, Park JY; The Korean Meta-Analysis (KORMA) Study Group. Efficacy of pharmacotherapy for smoking cessation in adolescent smokers: a meta-analysis of randomized controlled trials. Nicotine Tob Res. Epub 2018 Aug 18.

25. Xu FL, Wu X, Zhang JJ, Wang BJ, Yao J. A meta-analysis of data associating DRD4 gene polymorphisms with schizophrenia. Neuropsychiatr Dis Treat. 2018;14:153-164.

26. Zintzaras E, Ioannidis JP. Heterogeneity testing in meta-analysis of genome searches. Genet Epidemiol. 2005;28(2):123-137.

27. Higgins JP, Thompson SG, Deeks JJ, Altman DG. Measuring inconsistency in meta-analyses. BMJ. 2003;327(7414):557-560.

28. Naing C, Reid SA, Aung K. Comparing antibiotic treatment for leptospirosis using network meta-analysis: a tutorial. BMC Infect Dis. 2017; 17(1):29.

29. Sedgwick P, Marston L. How to read a funnel plot in a meta-analysis. BMJ. 2015;351:h4718.

30. Moher D, Liberati A, Tetzlaff J, Altman DG; PRISMA Group. Preferred reporting items for systematic reviews and meta-analyses: the PRISMA statement. PLoS Med. 2009;6(7):e1000097.

31. Konneker TI, Crowley JJ, Quackenbush CR, et al. No association of the serotonin transporter polymorphisms 5-HTTLPR and RS25531 with schizophrenia or neurocognition. Am J Med Genet B Neuropsychiatr Genet. 2010;153B(5):1115-1117.

32. Lin H, Lei Y, Zhang B, Dai Z, Lu X. Common variants of HTRIA and SLC6A4 confer the increasing risk of Schizophrenia susceptibility: A population-based association and epistasis analysis. Am JMed Genet Part B Neuropsychiatr Genet. 2015;168(8):749-755.

33. Chotai J, Serretti A, Lattuada E, Lorenzi C, Lilli R. Gene-environment interaction in psychiatric disorders as indicated by season of birth variations in tryptophan hydroxylase (TPH), serotonin transporter (5-HTTLPR) and dopamine receptor (DRD4) gene polymorphisms. Psychiatry Res. 2003;119(1-2):99-111.

34. Frisch A, Finkel B, Michaelovsky E, Sigal M, Laor N, Weizman R. A rare short allele of the serotonin transporter promoter region (5-HTTLPR) found in an aggressive schizophrenic patient of Jewish Libyan origin. Psychiatr Genet. 2000;10(4):179-183.

35. Dutt A, McDonald C, Dempster E, et al. The effect of COMT, BDNF, 5-HTT, NRG1 and DTNBP1 genes on hippocampal and lateral ventricular volume in psychosis. Psychol Med. 2009;39(11): $1783-1797$

36. Pae CU, Artioli P, Serretti A, et al. No evidence for interaction between 5-HT2A receptor and serotonin transporter genes in schizophrenia. Neurosci Res. 2005;52(2):195-199.

37. Sáiz PA, García-Portilla MP, Arango C, et al. Association study of serotonin 2A receptor (5-HT2A) and serotonin transporter (5-HTT) gene polymorphisms with schizophrenia. Prog Neuropsychopharmacol Biol Psychiatry. 2007;31(3):741-745.

38. Naylor L, Dean B, Pereira A, Mackinnon A, Kouzmenko A, Copolov D. No association between the serotonin transporter-linked promoter region polymorphism and either schizophrenia or density of the serotonin transporter in human hippocampus. Mol Med. 1998;4(10): 671-674.

39. Ikeda M, Iwata N, Suzuki T, et al. No association of serotonin transporter gene (SLC6A4) with schizophrenia and bipolar disorder in Japanese patients: association analysis based on linkage disequilibrium. J Neural Transm. 2006;113(7):899-905. 
40. Vijayan NN, Iwayama Y, Koshy LV, et al. Evidence of association of serotonin transporter gene polymorphisms with schizophrenia in a South Indian population. J Hum Genet. 2009;54(9):538-542.

41. Lee HY, Kim DJ, Lee HJ, Choi JE, Kim YK. No association of serotonin transporter polymorphism (5-HTTVNTR and 5-HTTLPR) with characteristics and treatment response to atypical antipsychotic agents in schizophrenic patients. Prog Neuropsychopharmacol Biol Psychiatry. 2009;33(2):276-280.

42. Pae CU, Kim JJ, Lee SJ, et al. Polymorphism of the serotonin transporter gene and symptomatic dimensions of schizophrenia in the Korean population. Neuropsychobiology. 2003;47(4):182-186.

43. Gu H, Liu C, Liu C, et al. The combined effects of the 5- HTTLPR and HTR1A rs6295 polymorphisms modulate decision making in schizophrenia patients. Genes Brain Behav. 2013;12(1):133-139.

44. Herken H, Erdal ME, Böke O, Savaş HA, Böke Ömer, Savas HA. Tardive dyskinesia is not associated with the polymorphisms of 5-HT2A receptor gene, serotonin transporter gene and catechol-omethyltransferase gene. Eur Psychiatry. 2003;18(2):77-81.

45. Serretti A, Lilli R, Lorenzi C, Lattuada E, Cusin C, Smeraldi E. Serotonin transporter gene (5-HTTLPR) and major psychoses. Mol Psychiatry. 2002;7(1):95-99.

46. Han DH, Park DB, Na C, Kee BS, Lee YS. Association of aggressive behavior in Korean male schizophrenic patients with polymorphisms in the serotonin transporter promoter and catecholamine-O-methyltransferase genes. Psychiatry Res. 2004;129(1):29-37.

47. Zaboli G, Jönsson EG, Gizatullin R, De Franciscis A, Åsberg M, Leopardi R. Haplotype analysis confirms association of the serotonin transporter (5-HTT) gene with schizophrenia but not with major depression. Am J Med Genet Part B Neuropsychiatr Genet. 2008;147B(3):301-307.

48. Golimbet V, Korovaitseva G, Lezheiko T, Abramova LI, Kaleda VG. The serotonin transporter gene 5-HTTLPR polymorphism is associated with affective psychoses but not with schizophrenia: A large-scale study in the Russian population. J Affect Disord. 2017;208:604-609.

49. Peitl V, Štefanović M, Karlović D. Depressive symptoms in schizophrenia and dopamine and serotonin gene polymorphisms. Prog Neuropsychopharmacol Biol Psychiatry. 2017;77:209-215.

50. Tea T, Matej K, Vita D, Kores PB. Influence of 5-HT1A and 5-HTTLPR genetic variants on the schizophrenia symptoms and occurrence of treatment-resistant schizophrenia. Neuropsychiatr Dis Treat. 2015;11: 453-459.

51. Golimbet VE, Korovaitseva GI, Faktor MI, Ganisheva TK, Dmitriev DA. Funktsional'nyy faktor serotoninergicheskoy sistemy i polimorfizm 5-HTTLPR gena serotoninovogo transportera u bol'nykh shizofreniyey [Functional siate of serotoninergic system and the 5-HTTLPR polymorphism of the serotonin transporter gene in patients with schizophrenia]. Mol Biol. 2010;44(2):251-256.

52. Golimbet VE, Alfimova MV, Shcherbatikh T, Kaleda VG, Abramova LI, Rogaev EI. Serotonin transporter gene polymorphism and schizoid personality traits in the patients with psychosis and psychiatrically well subjects. World J Biol Psychiatry. 2003;4(1):25-29.

53. Stöber G, Jatzke S, Heils A, et al. Susceptibility for schizophrenia is not influenced by a functional insertion/deletion variant in the promoter of the serotonin transporter gene. Eur Arch Psychiatry Clin Neurosci. 1998;248(2):82-86.

54. Kotler M, Barak P, Cohen H, et al. Homicidal behavior in schizophrenia associated with a genetic polymorphism determining low catechol O-methyltransferase (COMT) activity. Am J Med Genet. 1999;88(6): 628-633.

55. Rao D, Jönsson EG, Paus S, Ganguli R, Nöthen M, Nimgaonkar VL. Schizophrenia and the serotonin transporter gene. Psychiatr Genet. 1998;8(4):207-212.

56. Pakhomova SA, Korovă̌tseva GI, Miu M, et al. Molekulyarnogeneticheskiy izucheniye ranney shizofrenii [Molecular-genetic study of early-onset schizophrenia]. Zh Nevrol Psikhiatr Im S Korsakova. 2010;110(2):66-69. Russian.
57. Mendes de Oliveira JR, Otto PA, Vallada H, et al. Analysis of a novel functional polymorphism within the promoter region of the serotonin transporter gene (5-HTT) in Brazilian patients affected by bipolar disorder and schizophrenia. Am J Med Genet. 1998;81(3):225-227.

58. Bayle FJ, Leroy S, Gourion D, et al. 5HTTLPR polymorphism in schizophrenic patients: Further support for association with violent suicide attempts. Am J Med Genet Part B Neuropsychiatr Genet. 2003;119B(1): $13-17$.

59. Mata I, Arranz MJ, Patiño A, et al. Serotonergic polymorphisms and psychotic disorders in populations from North Spain. Am J Med Genet Part B Neuropsychiat Genet. 2004;126B(1):88-94.

60. Sanjuan J, Rivero O, Aguilar EJ, et al. Serotonin transporter gene polymorphism (5-HTTLPR) and emotional response to auditory hallucinations in schizophrenia. Int J Neuropsychopharmacol. 2006;9(1):131-133.

61. Chong SA, Lee WL, Tan CH, Tay AH, Chan AO, Tan EC. Attempted suicide and polymorphism of the serotonin transporter gene in Chinese patients with schizophrenia. Psychiatry Res. 2000;97(2-3):101-106.

62. Pae CU, Serretti A, Artioli P, et al. Interaction analysis between 5-HTTLPR and TNFA -238/-308 polymorphisms in schizophrenia. J Neural Transm. 2006;113(7):887-897.

63. Young KA, Holcomb LA, Bonkale WL, Hicks PB, Yazdani U, German DC. 5HTTLPR polymorphism and enlargement of the pulvinar: unlocking the backdoor to the limbic system. Biol Psychiatry. 2007; 61(6):813-818.

64. Jing Xuxiu ZR, Kerun G, Shibang S. Study on the correlation between the polymorphism in promoter region of 5-hydroxytryptamine transporter gene and schizophrenia in Chinese Han population. J Shanghai Jiaotong Univ. 2016;36(4):5.

65. Li ZM, Li W. A study of correlation between serotonin transporter gene polymorphism and schizophrenia. J Clin Psychiatry. 2013;23(6):4.

66. Zuo Sue WX, Guiqing C, Yichang C, Yong S. Analysis of the correlation between 5-H T T L P R and mental splitting attack behavior. Chin J Forensic Med. 2013;18(2):2.

67. Tsai SJ, Hong CJ, Yu YW, et al. Association study of a functional serotonin transporter gene polymorphism with schizophrenia, psychopathology and clozapine response. Schizophr Res. 2000;44(3):177-181.

68. Lu AT, Bakker S, Janson E, Cichon S, Cantor RM, Ophoff RA. Prediction of serotonin transporter promoter polymorphism genotypes from single nucleotide polymorphism arrays using machine learning methods. Psychiatr Genet. 2012;22(4):182-188.

69. Bonnet-Brilhault F, Laurent C, Thibaut F, et al. Serotonin transporter gene polymorphism and schizophrenia: an association study. Biol Psychiatry. 1997;42(7):634-636.

70. Liu W, Gu N, Feng G, et al. Tentative association of the serotonin transporter with schizophrenia and unipolar depression but not with bipolar disorder in Han Chinese. Pharmacogenetics. 1999;9(4):491-495.

71. Lin C, Tang W, Hu J, et al. Haplotype analysis confirms association of the serotonin transporter (5-HTT) gene with schizophrenia in the Han Chinese population. Neurosci Lett. 2009;453(3):210-213.

72. Tsai SJ, Ouyang WC, Hong CJ. Association for serotonin transporter gene variable number tandem repeat polymorphism and schizophrenic disorders. Neuropsychobiology. 2002;45(3):131-133.

73. Herken H, Erdal ME, Aynacioğlu AS, et al. Frequency of the 17-bp variable number of tandem repeat polymorphism in Turkish schizophrenic patients. Schizophr Res. 2002;58(1):99-100.

74. Collier DA, Arranz MJ, Sham P, et al. The serotonin transporter is a potential susceptibility factor for bipolar affective disorder. Neuroreport. 1996;7(10):1675-1679.

75. Yang S. Correlation analysis between SERT gene and schizophrenia and analysis of its single nucleotide polymorphism [master's thesis]. Kirkland: Northwest University; 2001. Chinese.

76. Lindholm Carlström E, Saetre P, Rosengren A, et al. Association between a genetic variant in the serotonin transporter gene (SLC6A4) and suicidal behavior in patients with schizophrenia. Behav Brain Funct. 2012;8(1):24. 
77. Xuan JF, Ding M, Pang H, et al. [Relationship between genetic polymorphisms of 3 SNP loci in 5-HTT gene and paranoid schizophrenia]. Fa Yi Xue Za Zhi. 2012;28(6):418-421. Chinese.

78. Li W, Yang Y, Lin J, et al. Association of serotonin transporter gene (SLC6A4) polymorphisms with schizophrenia susceptibility and symptoms in a Chinese-Han population. Prog Neuropsychopharmacol Biol Psychiatry. 2013;44:290-295.

79. Pal P, Mihanović M, Molnar S, et al. Association of tagging single nucleotide polymorphisms on 8 candidate genes in dopaminergic pathway with schizophrenia in Croatian population. Croatian Med J. 2009;50(4):361-369

80. Qi XL, Xuan JF, Xing JX, Wang BJ, Yao J. No association between dopamine D3 receptor gene Ser9Gly polymorphism (rs6280) and risk of schizophrenia: an updated meta-analysis. Neuropsychiatr Dis Treat. 2017; 13:2855-2865

81. Palmer SC, Gardner S, Tonelli M, et al. Phosphate-Binding Agents in Adults With CKD: A Network Meta-analysis of Randomized Trials. Am J Kidney Dis. 2016;68(5):691-702.

82. Thakkinstian A, McElduff P, D'Este C, Duffy D, Attia J. A method for meta-analysis of molecular association studies. Stat Med. 2005;24(9): 1291-1306.

83. Fanelli G, Serretti A. The influence of the serotonin transporter gene 5-HTTLPR polymorphism on suicidal behaviors: a meta-analysis. Prog Neuropsychopharmacol Biol Psychiatry. 2019;88:375-387.

84. Hu XZ, Lipsky RH, Zhu G, et al. Serotonin transporter promoter gainof-function genotypes are linked to obsessive-compulsive disorder. Am J Hum Genet. 2006;78(5):815-826.

85. Rao S, Han X, Shi M, et al. Associations of the serotonin transporter promoter polymorphism (5-HTTLPR) with bipolar disorder and treatment response: A systematic review and meta-analysis. Prog Neuropsychopharmacol Biol Psychiatry. 2019;89:214-226.

86. Wendland JR, Moya PR, Kruse MR, et al. A novel, putative gain-offunction haplotype at SLC6A4 associates with obsessive-compulsive disorder. Hum Mol Genet. 2008;17(5):717-723.

87. Hranilovic D, Stefulj J, Schwab S, et al. Serotonin transporter promoter and intron 2 polymorphisms: relationship between allelic variants and gene expression. Biol Psychiatry. 2004;55(11):1090-1094.

88. Lovejoy EA, Scott AC, Fiskerstrand CE, Bubb VJ, Quinn JP. The serotonin transporter intronic VNTR enhancer correlated with a predisposition to affective disorders has distinct regulatory elements within the domain based on the primary DNA sequence of the repeat unit. Eur J Neurosci. 2003;17(2):417-420.

89. Fiskerstrand CE, Lovejoy EA, Quinn JP. An intronic polymorphic domain often associated with susceptibility to affective disorders has allele dependent differential enhancer activity in embryonic stem cells. FEBS Lett. 1999;458(2):171-174.
90. Wang KS, Liu XF, Aragam N. A genome-wide meta-analysis identifies novel loci associated with schizophrenia and bipolar disorder. Schizophr Res. 2010;124(1-3):192-199.

91. Zhao Y, Liang X, Zhu F, et al. A large-scale integrative analysis of GWAS and common meQTLs across whole life course identifies genes, pathways and tissue/cell types for three major psychiatric disorders. Neurosci Biobehav Rev. 2018;95:347-352.

92. Ikeda M, Takahashi A, Kamatani Y, et al. Genome-Wide Association Study Detected Novel Susceptibility Genes for Schizophrenia and Shared Trans-Populations/Diseases Genetic Effect. Schizophrenia Bulletin. 2018;511.

93. Yin L, Cheung EF, Chen RY, Wong EH, Sham PC, So HC. Leveraging genome-wide association and clinical data in revealing schizophrenia subgroups. J Psychiatr Res. 2018;106:106-117.

94. Jagannathan K, Calhoun VD, Gelernter J, et al. Genetic associations of brain structural networks in schizophrenia: a preliminary study. Biol Psychiatry. 2010;68(7):657-666.

95. Frey S. The economic burden of schizophrenia in Germany: a population-based retrospective cohort study using genetic matching. Eur Psychiatry. 2014;29(8):479-489.

96. Walton E, Liu J, Hass J, et al. $M B-C O M T$ promoter DNA methylation is associated with working-memory processing in schizophrenia patients and healthy controls. Epigenetics. 2014;9(8):1101-1107.

97. Yao J, Pan YQ, Ding M, Pang H, Wang BJ. Association between DRD2 (rs1799732 and rs1801028) and ANKK1 (rs1800497) polymorphisms and schizophrenia: a meta-analysis. Am JMed Genet B Neuropsychiatr Genet. 2015;168B(1):1-13.

98. Correa H, Campi-Azevedo AC, de Marco L, et al. Familial suicide behaviour: association with probands suicide attempt characteristics and 5-HTTLPR polymorphism. Acta Psychiatr Scand. 2004;110(6): 459-464.

99. Semwal P, Prasad S, Bhatia T, et al. Family-based association studies of monoaminergic gene polymorphisms among North Indians with schizophrenia. Mol Psychiatry. 2001;6(2):220-224.

100. Dubertret C, Hanoun N, Adès J, Hamon M, Gorwood P. Family-based association study of the 5-HT transporter gene and schizophrenia. Int J Neuropsychopharmacol. 1999;8(1):87-92.

101. Dimitrova A, Georgieva L, Nikolov I, et al. Major psychiatric disorders and the serotonin transporter gene (SLC6A4): family-based association studies. Psychiatr Genet. 2002;12(3):137-141.
Neuropsychiatric Disease and Treatment

\section{Publish your work in this journal}

Neuropsychiatric Disease and Treatment is an international, peerreviewed journal of clinical therapeutics and pharmacology focusing on concise rapid reporting of clinical or pre-clinical studies on a range of neuropsychiatric and neurological disorders. This journa is indexed on PubMed Central, the 'PsycINFO' database and CAS,

\section{Dovepress}

and is the official journal of The International Neuropsychiatric Association (INA). The manuscript management system is completely online and includes a very quick and fair peer-review system, which is all easy to use. Visit http://www.dovepress.com/testimonials.php to read real quotes from published authors. 